\title{
The Relationship of Salicylate Lipophilicity to Rectal Insulin Absorption Enhance- ment and Relative Lymphatic Uptake
}

\author{
David J. HAUSS, ${ }^{*}$ Howard Y. ANDO, ${ }^{* *}$ Dennis M. GRASELA, ${ }^{* *}$ and Edwin T. SUGITA \\ Department of Pharmaceutics, The Philadelphia College of Pharmacy \& Science, Philadelphia, \\ PA 19104, U.S.A.
}

(Received September 17, 1990)

\begin{abstract}
The sodium salts of the 3,5-dichloro, 3,5-dibromo-, 3,5-diiodo-, and 5-methoxy- analogs of salicylic acid have been evaluated as enhancers of rectal insulin absorption. A relationship was found between adjuvant potency and relative lipophilicity. Maximal adjuvant activity was obtained with $0.1 \mathrm{~m}$ 3,5-dichlorosalicylate. Higher concentrations $(0.15 \mathrm{M})$ of 3,5-diiodosalicylate produced a decline in adjuvant activity, which may be associated with extraction of specific cellular proteins. This may indicate the existence of an optimal salicylate lipophilicity for adjuvant efficacy. Relative adjuvant activity was found to be related to the lymph:plasma absorption ratio of $\left[{ }^{125} \mathrm{I}\right]$ insulin. Lymphatic uptake of insulin was not related to lymph flow rate.
\end{abstract}

Keywords — adjuvant; insulin; salicylate; rectal absorption; lymphatic; [ $\left.{ }^{125} \mathrm{I}\right]$ insulin

\section{Introduction}

Previous work performed in our laboratories suggested the existence of a relationship between the concentration of two salicylate derivatives, 5-methoxysalicylate sodium (MS) and 3,5-diiodosalicylate sodium (DIS), and their ability to enhance rectal insulin absorption. ${ }^{1}$ Adjuvant potency also appeared to be related to lipophilicity. 1) Earlier studies conducted by Levitan and Barker on the effects of salicylates on membrane ionic permeability enhancement revealed a similar relationship between potency and salicylate lipophilicity. ${ }^{2)}$ To further evaluate the relationship between adjuvant lipophilicity and potency, two additional salicylate analogs of different lipophilicities, 3,5-dibromosalicylate sodium and 3,5-dichlorosalicylate sodium, have been studied.

In a previous study, we demonstrated that high concentrations of DIS were associated with a decline in adjuvant activity. "In an attempt to elucidate the mechanism of decreased insulin $a b$ sorption seen at high concentrations of DIS, we investigated the relationship of salicylate adjuvant activity to degree and specificity of mem- brane protein extraction. DIS is an efficient solubilizer of membrane proteins. ${ }^{3)}$ Various investigators have studied the relationship between adjuvant activity and membrane protein extraction. Adjuvants whose absorption-enhancing activities are associated with major histological changes in epithelial membranes (i.e., anionic surfactants, bile salts) show correlations between degree of adjuvant activity and magnitude of membrane protein extraction for both in vivo and in vitro preparations. A decline in adjuvant activity was seen at high concentrations of sodium deoxycholate and was found to be associated with protein extraction. ${ }^{4)}$ Similarly, elevated concentrations of the adjuvant diethyl maleate (DEM) exhibited decreased enhancement of cefmetazole absorption from rat ileal loops; the diminished adjuvant activity, in this instance, was associated with extraction of membrane protein thiol. ${ }^{5)}$ In vitro extraction of protein from everted rat rectal sacs by sodium salicylate, a relatively weak adjuvant in comparison to DIS, was reported to be not significantly different from control. ${ }^{6}$ To our knowledge, no work has been published on the in vivo extraction of membrane proteins by salicylate adjuvants.

\footnotetext{
* Present Address: Parke-Davis Pharmaceutical Research, 170 Tabor Road, Morris Plains, New Jersey 07950, U.S.A.

** To whom reprint requests should be addressed.

*** Present Address: The Squibb Institute for Medical Research, P.O. Box 4000, Princeton, N. J. 08543-4000, U.S.A.
} 
Nadai et al. ${ }^{7)}$ showed that elevated concentrations of the adjuvants, tetracycline and ethylenediamine tetra acetic acid (EDTA), were associated with declines in relative lymphatic absorption of sulfanilic acid from the rat small intestine. To test the hypothesis that adjuvant activity might be related to the relative degree of lymphatic targeting of insulin by the adjuvants, the limph:blood absorption ratios for $\left[{ }^{125} \mathrm{I}\right] \mathrm{insu}$ lin were determined at serial concentrations of each adjuvant.

The purposes of this study are: 1) to further evaluate the relationship between adjuvant lipophilicity and potency by studying two additional salicylate analogs of different lipophilicities; 2 ) to evaluate the possibility of a relationship between salicylate adjuvant activity and degree and/or specificity of membrane protein extraction; 3$)$ to investigate the effects of concentration and salicylate lipophilicity on targetting of [ $\left.{ }^{125} \mathrm{I}\right]$ insulin to the lymphatic compartment.

\section{Materials and Methods}

Chemicals — Human insulin injection, USP (Eli Lilly, Indianapolis, IN) was used. The 5-methoxy-, 3,5-dichloro-, 3,5-dibromo-, and 3,5-diiodo- analogs of salicylic acid were obtained from Aldrich Chemical Company, Milwaukee, WI, and converted to the sodium salts by titration with sodium hydroxide in absolute ethanol. ${ }^{2)}\left[{ }^{125} \mathrm{I}\right]$ Insulin (sp. act. $140 \mu \mathrm{Ci} / \mu \mathrm{g}$ ) was obtained from Cambridge Medical Diagnostics (Billerica, MA). Electrophoresis grade acrylamide and $N, N^{\prime}$-bismethylene acrylamide were purchased from Bio-Rad Labs (Rockville Centre, NY). Other reagents used for electrophoresis were of molecular biology grade.

Preparation of Microenema Solutions Microenema solutions were freshly prepared by dissolving the appropriate amount of adjuvant in a vehicle consisting of $0.2 \mathrm{M}$ phosphate buffer (pH 5) containing 50\% propylene glycol, which was necessary to solubilize the more lipophilic adjuvants. Insulin was then added to a final concentration of $6 \mathrm{I} . \mathrm{U} . / \mathrm{ml}$ of solution. For the lymph:blood partitioning studies, microenema solutions were prepared as above. Prior to administration, $2 \mu \mathrm{Ci}$ of $\left[{ }^{125} \mathrm{I}\right]$ insulin was added to each microenema.

Insulin Absorption Studies - Relative enhancement of insulin absorption was determined as described in a previous paper. ${ }^{1)}$ Briefly, male Sprague-Dawley rats (200-300 g) from Taconic Farms (Germantown, NY) were fasted for $20 \mathrm{~h}$ before experiments for which they were anesthetized with pentobarbital sodium $(50 \mathrm{mg} / \mathrm{kg})$. The right external jugular vein was cannulated and serial blood samples $(100 \mu \mathrm{l})$ were drawn immediately before and at 15, 30, 60, 90, and $120 \mathrm{~min}$ following the microenema. Glucose determinations were made on $20 \mu \mathrm{l}$ volumes of blood using a commercially available kit (Accu-Check II, Boehringer Mannheim Diagnostics Division, Indianapolis, IN).

Sodium Dodecyl Sulfate-Polyacrylamide Gel Electrophoresis (SDS-PAGE) of SalicylateExtracted Membrane Proteins - Microenema solutions of each of the adjuvants were prepared as described above, with the exception that insulin was omitted. A control solution was prepared consisting of $0.2 \mathrm{M}$ sodium phosphate buffer (pH 5) and 50\% propylene glycol. For the in vivo studies, male Sprague-Dawley rats $(200-300 \mathrm{~g})$ were fasted for $20 \mathrm{~h}$ prior to experiments for which they were anesthetized with pentobarbital sodium $(50 \mathrm{mg} / \mathrm{kg})$ and the abdomen opened with a $6 \mathrm{~cm}$ midline incision. A 7 $\mathrm{cm}$ segment of the distal colon was isolated on two 4-0 silk ligatures which were tightened firmly so as to form a closed sac; care was taken not to ligate the hemorrhoidal vessels. One $\mathrm{ml}$ of the adjuvant solution was injected into the lumen of the intestinal sac with a tuberculin syringe. After $1 \mathrm{~h}$, the solution was removed with a second syringe. These solutions were dialyzed in 12000 - 14000 dalton (Da) cut-off dialysis bags (Pope Scientific, Menomonee Falls, WI) against $0.0625 \mathrm{M}$ Tris buffer ( $\mathrm{pH} \mathrm{8.8)}$ for $24 \mathrm{~h}$ at $4{ }^{\circ} \mathrm{C}$ in a Pope Microdialyzer, model II. This served to remove salicylate and propylene glycol, both of which interfere with the protein assay and electrophoresis.

In vitro protein extraction was determined using an everted gut technique. Four centimeter sections of distal colon were removed from male Sprague-Dawley rats, anesthetized as above, and everted with a $3 \mathrm{~mm}$ glass rod. Both ends of the 
segments were tied firmly with $3-0$ silk so as to form small sacs, which were then placed into 7 $\mathrm{ml}$ glass vials containing $5 \mathrm{ml}$ of Krebbs-Ringer bicarbonate buffer solution (KRBB) $(\mathrm{pH} 7.4)$ that had been thoroughly gassed with 95:5 $\mathrm{O}_{2}: \mathrm{CO}_{2}$. Control samples contained KRBB only; treated samples contained, in addition, either DIS $0.025 \mathrm{M}$ or MS $0.15 \mathrm{M}$. All samples were prepared in triplicate. After a $1 \mathrm{~h}$ incubation at $37{ }^{\circ} \mathrm{C}$, the solutions were removed and dialyzed, as above, against $0.0625 \mathrm{M}$ Tris $(\mathrm{pH}$ 8.8) for $24 \mathrm{~h}$ at $4{ }^{\circ} \mathrm{C}$.

Total protein concentration was determined with the Pierce BCA protein assay (Pierce Chemical Company, Rockford, IL). One-hundred microliter amounts of dialysate were incubated with $2 \mathrm{ml}$ of the BCA protein assay reagent in $4.5 \mathrm{ml}$ polystyrene cuvettes (Curtin Matheson Scientific, Houston, TX) at $37^{\circ} \mathrm{C}$ for $30 \mathrm{~min}$. Those samples for which total protein concentration exceeded $1200 \mu \mathrm{g}$ were diluted 1:10 with distilled water and $100 \mu \mathrm{l}$ of the resulting dilution assayed.

Eight-to-25\% linear concentration gradient polyacrylamide slab gels, $14 \mathrm{~cm} \times 16 \mathrm{~cm} \times 1.5$ $\mathrm{mm}$ were prepared from solutions of $8 \%$ and $25 \%$ by weight of acrylamide and $0.26 \%$ and $1.54 \%$ of $N, N^{\prime}$-bismethylene acrylamide (J. R. Porter, Personal Communication). The final concentrations in the separating gel were as follows: $0.375 \mathrm{M}$ Tris- $\mathrm{HCl}(\mathrm{pH}$ 8.8) $0.1 \%$ SDS and $0.3 \%$ ammonium persulfate. Quantities of 15.5 $\mathrm{ml}$ of each of the solutions were placed in the wells of a Hoefer SG50 gradient former (Hoefer Scientific Instruments, San Francisco, CA) and TEMED added, $3 \mu$ lo the $25 \%$ acrylamide solution and $7 \mu \mathrm{l}$ to the $8 \%$ solution, and the gel was poured over $5 \mathrm{~min}$. Following polymerization, the separating gel was overlayed with a stacking gel prepared from $3 \%$ by weight of acrylamide and $0.1 \%$ by weight of $N, N^{\prime}$-bismethylene acrylamide. The final concentrations in the stacking gel were: $0.375 \mathrm{M}$ Tris- $\mathrm{HCl}(\mathrm{pH}$ $8.8), 0.1 \%$ SDS, $0.12 \%$ ammonium persulfate, and sucrose $5 \%$. The stacking gel was polymerized chemically by addition of $10 \mu \mathrm{l}$ of TEMED.

The electrode buffer contained $0.025 \mathrm{M}$ Tris$\mathrm{HCl}(\mathrm{pH} \mathrm{8.3)}, 0.192 \mathrm{M}$ glycine, and $0.1 \%$ SDS. The samples were prepared by diluting $400 \mu \mathrm{l}$ volumes of sample with an equal volume of Seprasol (Integrated Separation Systems, Hyde Park, MA) and contained the following concentrations: $0.0625 \mathrm{M}$ Tris- $\mathrm{HCl}(\mathrm{pH} 6.8), 2 \%$ SDS, $5 \%$ sucrose, $5 \%$ 2-mercaptoethanol, and $0.001 \%$ bromphenol blue as the tracking dye. The proteins were completely dissociated by immersing the samples for $5 \mathrm{~min}$ in a boiling water bath. Gels were loaded with $25 \mu \mathrm{g}$ quantities of sample proteins and electrophoresis carried out in a Biorad Protean I electrophoresis chamber at an initial current of 20 ma per gel, raised to $30 \mathrm{ma}$ per gel once the tracking dye had entered the resolving gel. Electrophoresis was continued until the tracking dye reached $1 \mathrm{~cm}$ from the bottom of the gel. Proteins were revealed with the Bio Rad silver stain kit. Molecular weights of unknown proteins were determined relative to a series of marker proteins ranging in weight from 12400 to 95500 Da (Mid Range Kit, Diversified Biotech, Newton Centre, MA).

Transmittance densitometric scans of the gels were conducted on a Bio-Rad model 1650 densitometer at a scan speed of $7.5 \mathrm{~cm} / \mathrm{min}$ and plots made on a Spectra-Physics SP4290 integrator (Spectra-Physics, San Jose, CA).

Lymph:Blood Partitioning Studies — To assess the abilities of the various adjuvants to selectively target rectally absorbed insulin to the lymphatic compartment, absorption of [ $\left.{ }^{125} \mathrm{I}\right] \mathrm{in}$ sulin into blood and lymph was studied. For these studies, two concentrations of each adjuvant were chosen from the constructed linear portions (see Insulin Absorption Studies in Results section) of the respective log doseresponse curves. In addition, DIS $0.15 \mathrm{M}$ was also evaluated to determine if the decline in adjuvant activity at this concentration was related to a change in the lymph:blood partition ratio of insulin. ${ }^{5,7)}$ For these studies, animals were fasted and anesthetized as described above. The right external jugular vein was cannulated and the cannula exteriorized through a small hole in the back of the neck. Three $\mathrm{ml}$ of a 10 units $/ \mathrm{ml}$ solution of heparin in saline was rapidly administered via the jugular vein to aid in visualization of the thoracic lymph duct, which was then cannulated with PE 50 tubing. ${ }^{8)}$ Animals were transferred to Bollman restraining cages 
and permitted to recover. To prevent dehydration due to lymph loss, normal saline was infused continuously at $3.6 \mathrm{ml} / \mathrm{h}$ through a 4-way stopcock (Medical Surgical Concepts, Inc. No STS04PF) connected to the jugular venous cannula. Following microenema administration, the anus was ligated with thread to prevent leakage and lymph collected into tared $20 \mathrm{ml}$ glass scintillation vials as a function of time. The first four samples were collected over 15 min intervals; the fifth sample was collected over $30 \mathrm{~min}$. Lymph volume was determined by weighing. Fifteen $\mathrm{ml}$ of Ready-Safe scintillation cocktail (Beckman Instruments, Inc., Fullerton, CA) was added to each sample and thoroughly mixed by shaking. Blood samples $(300 \mu \mathrm{l})$ were drawn at the end of each collection interval. The stopcock was flushed with $400 \mu \mathrm{l}$ of a 10 units $/ \mathrm{ml}$ solution of heparin in normal saline. Blood samples were transferred into $1.5 \mathrm{ml}$ polypropylene centrifuge tubes heparinized by adding $20 \mu \mathrm{l}$ of a 20 units $/ \mathrm{ml}$ solution to the tubes and permitting it to evaporate. The samples were immediately centrifuged for $5 \mathrm{~min}$ in an Eppendorf 5414 centrifuge and $100 \mu \mathrm{l}$ volumes of plasma transferred into $7 \mathrm{ml}$ glass scintillation vials. Five $\mathrm{ml}$ of Ready-Safe scintillation cocktail was added to each sample and thoroughly vortexed. Radioactivity determinations were made using a Packard model 4530 liquid scintillation counter.

\section{Results}

\section{Insulin Absorption Studies}

The relative ability of the four adjuvants to enhance insulin absorption was determined from the maximum percent decrease in blood sugar from initial value $\left(E_{\max } \%\right) .{ }^{9)}$ The mean values of $E_{\max } \%$ ( \pm S.D.) as a function of adjuvant concentration are depicted in Fig. 1. $T_{\max }$
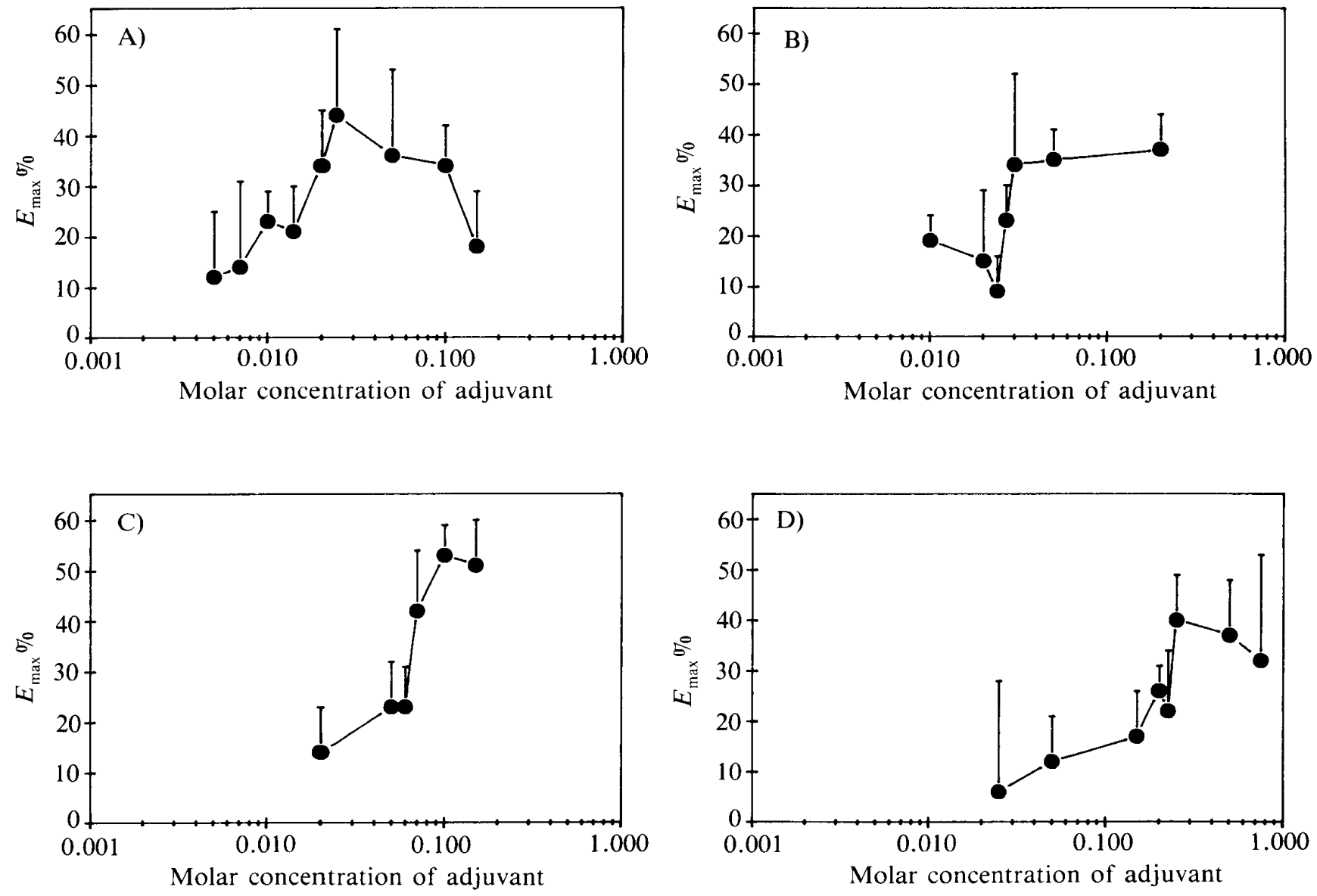

Fig. 1. Relative Adjuvant Activity, Expressed as the $E_{\max } \%$ Values, as a Function of Adjuvant Concentrations Data points represent the means \pm the standard deviations $(n \geq 4)$. A) DIS, B) DBS, C) DCS, D) MS. 
TABLE I. Relationship of Adjuvant Concentration to $E_{\max } \%$ and $T_{\max }$ Values

\begin{tabular}{|c|c|c|c|c|c|}
\hline \multicolumn{3}{|c|}{ DIS } & \multicolumn{3}{|c|}{ DBS } \\
\hline Conc. $_{\text {(molar) }}$ & $E_{\max } \%$ & $T_{\max }(\min )$ & Conc. $_{\text {(molar) }}$ & $E_{\max } \%$ & $T_{\max }(\min )$ \\
\hline 0.005 & 7.5 & 60 & 0.010 & 18.6 & 60 \\
\hline 0.007 & 13.9 & 60 & 0.020 & 15.1 & 30 \\
\hline 0.010 & 22.9 & 30 & 0.024 & 9.3 & 30 \\
\hline 0.014 & 18.3 & 30 & 0.027 & 21.6 & 30 \\
\hline 0.020 & 34.5 & 60 & 0.030 & 34.2 & 30 \\
\hline 0.024 & 44.4 & 90 & 0.050 & 35.0 & 60 \\
\hline 0.050 & 35.6 & 60 & 0.200 & 37.4 & 90 \\
\hline 0.100 & 33.6 & 60 & & & \\
\hline 0.150 & 18.1 & 60 & & & \\
\hline
\end{tabular}

\begin{tabular}{lcccccc}
\hline \hline & DCS & & & \multicolumn{3}{c}{ MS } \\
\cline { 1 - 3 } \cline { 6 - 7 } Conc. $_{\text {(molar) }}$ & $E_{\max } \%$ & $T_{\max }(\min )$ & & Conc. $_{(\text {molar })}$ & $E_{\max } \%$ & $T_{\max }(\min )$ \\
\hline 0.020 & 13.6 & 30 & & 0.025 & 6.3 & 30 \\
0.050 & 22.3 & 60 & & 0.050 & 12.2 & 30 \\
0.060 & 20.6 & 30 & & 0.150 & 16.8 & 30 \\
0.070 & 41.4 & 90 & & 0.200 & 25.8 & 30 \\
0.100 & 53.3 & 90 & & 0.224 & 26.1 & 30 \\
0.150 & 50.9 & 90 & & 0.250 & 40.0 & 60 \\
& & & 0.500 & 37.1 & 60 \\
& & & 0.750 & 31.6 & 60 \\
\hline
\end{tabular}

values (times at which $E_{\max } \%$ values were observed) are displayed in Table I, along with the respective $E_{\max } \%$ values and associated adjuvant concentrations. The maximum adjuvant effect was demonstrated by $0.1 \mathrm{M}$ DCS $(p<0.05$, $n=6)$. The decrease in the DIS $E_{\max } \%$ from its peak value at $0.05 \mathrm{M}$ is statistically significant $(p<0.05, n=6)$ at $0.15 \mathrm{M}$. The slight decline in adjuvant activities of DCS and MS at maximum concentration is not significant ( $p>0.05, n>4)$. A decline in the maximal degree of DIS adjuvant activity seen at $0.05 \mathrm{M}$ concentration, was noted for DIS $0.15 \mathrm{M}$ and was statistically significant $(p<0.05, n \geq 5)$. ${ }^{1)}$ A control experiment run on vehicle solutions containing $0.15 \mathrm{M}$ DIS and no insulin demonstrated no significant change in blood sugar from initial value, indicating that the decreased hypoglycemic effect seen at that concentration of DIS was probably not caused by an adjuvant-induced hyperglycemia $(p>0.05, n=3) .{ }^{1)}$ Control experiments run on vehicle solutions containing insulin $6 \mathrm{I} . \mathrm{U} . / \mathrm{ml}$ showed no decrease in blood sugar from initial

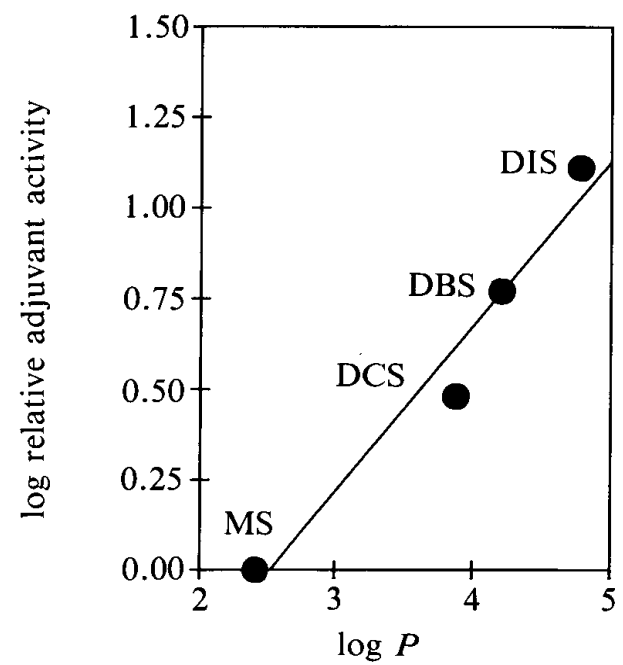

Fig. 2. Relationship of the Logarithm of Relative Adjuvant Activities Expressed as $E_{\max } \%$ Values to Relative Lipophilicities, Expressed as the Logarithm of the Octanol: Water Parititon Coefficients ${ }^{6)}$ 


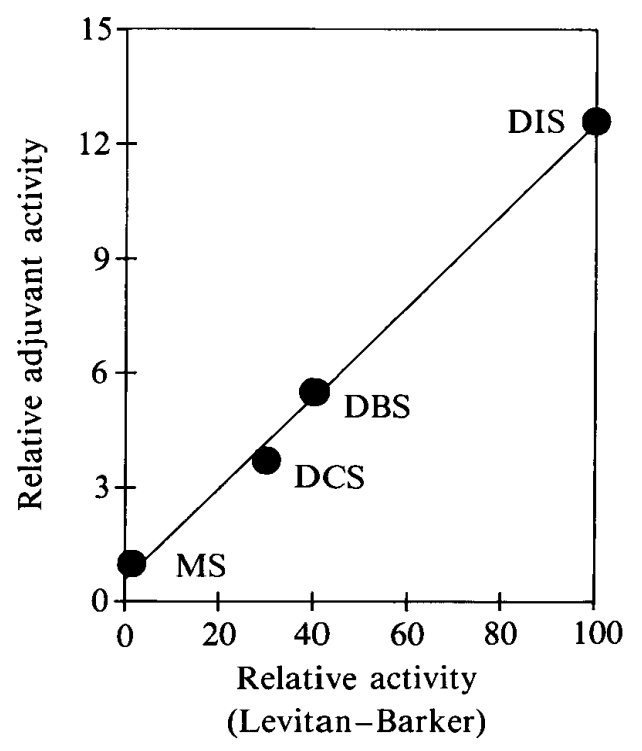

Fig. 3. Relationship of Relative Adjuvant Activities, Expressed as $E_{\max } \%$ Values, to Relative Effects on Membrane Ionic Permeability ${ }^{6}$

value $(p>0.05, n=3) .{ }^{1)}$ Relative adjuvant potency determinations were made by constructing parallel lines over the linear portions of the log dose-response curves and taking the antilog of the distance between these lines. ${ }^{10)}$ Linear portions of the curves were taken to be those points falling on that portion of the curve representing approximately $20 \%$ to $80 \%$ of $E_{\max } \%{ }^{10)}$ Due to the narrow concentration range existing between minimum and maximum response for DBS, points falling between $40 \%$ and $91 \%$ of maximum were used in determining the linear portion of the curve. Relative to MS, the adjuvants DCS, DBS, and DIS were respectively $3.7,5.5$, and 12.6 times more potent. A positive relationship existed between the log of the adjuvant octanol:water partition coefficient $(\log P)$ and the $\log$ of the relative potency (Fig. 2). Additionally, the relative potencies of these compounds as enhancers of insulin absorption was linearly related to their effectiveness as enhancers of membrane ionic permeability (Fig. 3). ${ }^{2)}$

\section{Limph:Blood Partitioning Studies}

Lymph:blood partition ratios for insulin were determined as the ratio of the corresponding areas of the lymph and plasma $\mathrm{dpm} / \mathrm{ml} v s$. time curves from 0 to $1.5 \mathrm{~h}$. This time interval was chosen since salicylate-enhanced absorption of rectally delivered insulin has been shown to be essentially complete at the end of this period. ${ }^{11-13)}$ For MS, the time of peak appearance of tracer in both lymph (Fig. 4) and plasma (Fig. 5) corresponded well with that of immunoreactive insulin ${ }^{12)}$ as well as with time of maximum hypoglycemic effect. Taken together, these observations infer that absorbed radioactivity during the 0 to $1.5 \mathrm{~h}$ time interval represents intact insulin. ${ }^{14)}$ A relationship was found to exist between relative adjuvant activity $\left(E_{\max } \%\right)$ and
A) MS $0.24 \mathrm{M}$

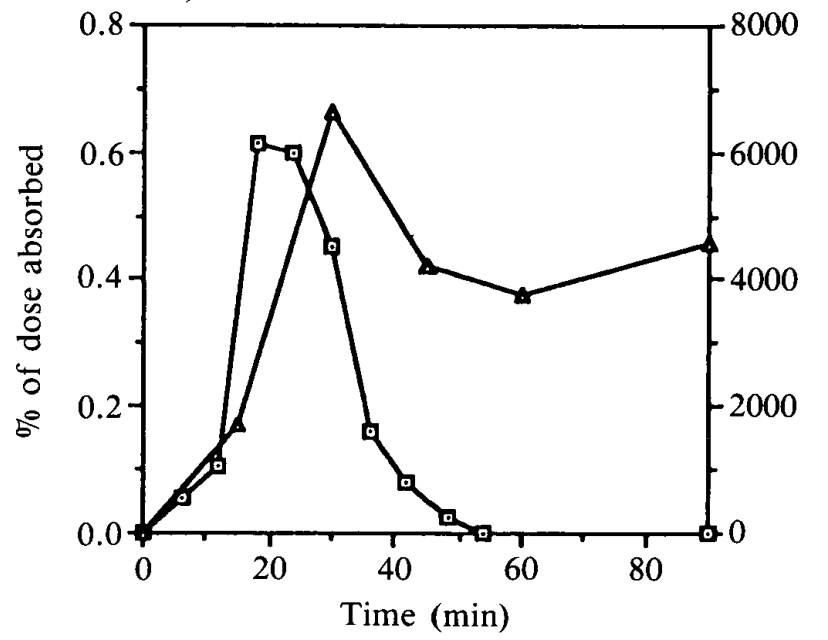

B) control

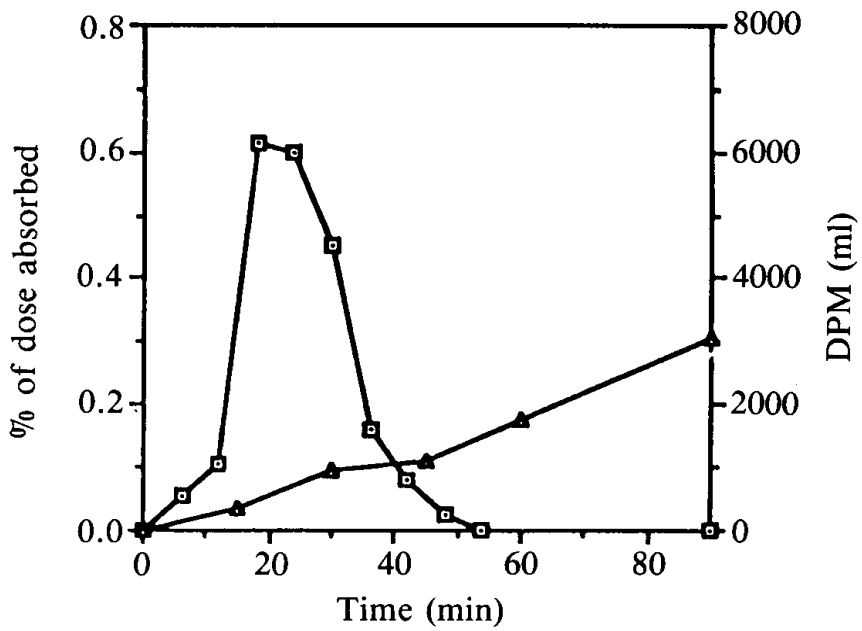

Fig. 4. Comparison of Time Course of Appearance of Radioactivity in Rat Thoracic Duct Lymph, as a Function of Time $(\triangle)$, with Appearance of Immunoreactive Insulin in Hepatic Portal Plasma $(\square)^{12)}$,*

A) MS 0.24 M (lymph), B) control (lymph). 
A) MS $0.24 \mathrm{M}$

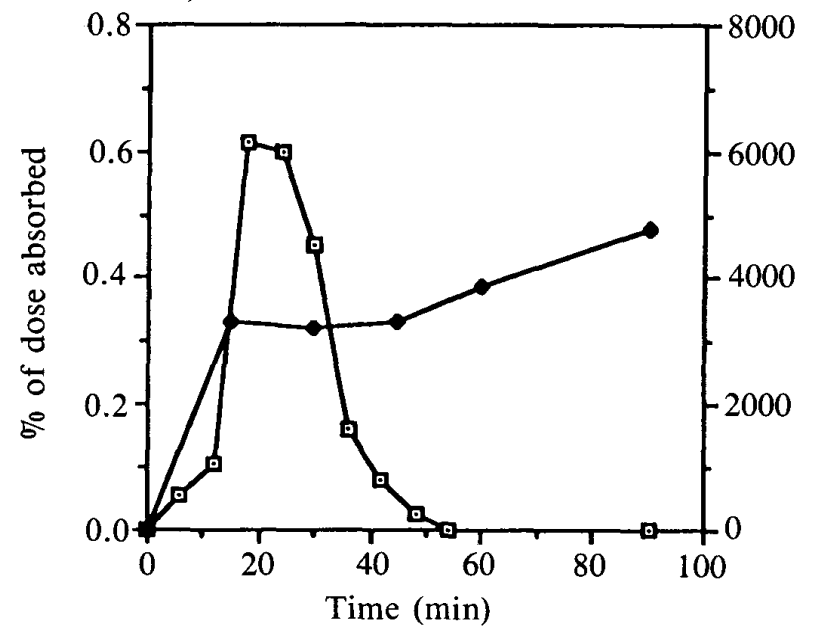

B) control

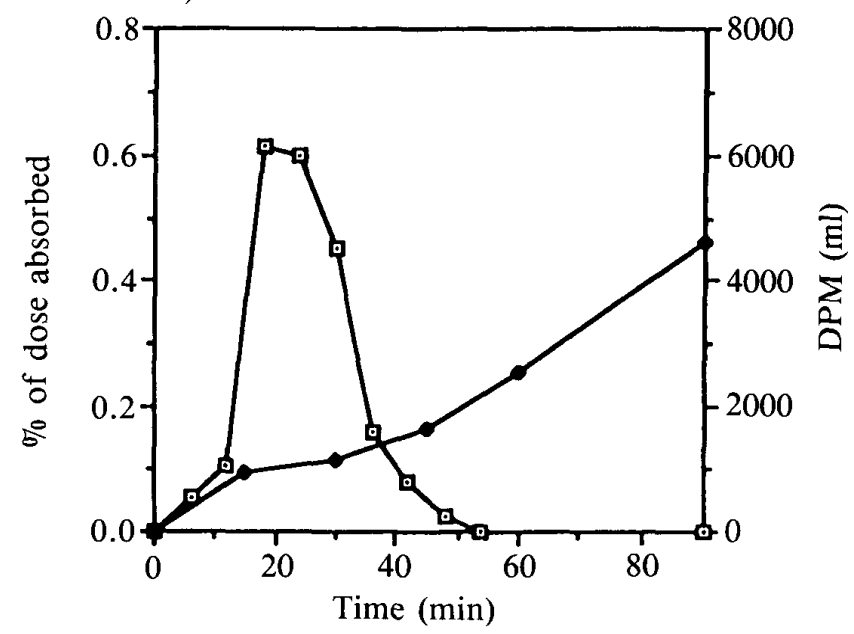

Fig. 5. Comparison of Time Course of Appearance of Radioactivity in Rat Plasma ( ), as a Function of Time, with Appearance of Immunoreactive Insulin in Hepatic Portal Plasma ( $\square)^{12)}$,*

A) MS 0.24 m (plasma), B) control (plasma).

the corresponding lymph:plasma partition ratio of [ ${ }^{125}$ I] insulin (Fig. 6). A linear relationship existed between extent of tracer absorption into lymph and adjuvant activity. Absorption of tracer into plasma was closely related to corresponding lymphatic absorption at values of $E_{\max } \%<34$. For values of $E_{\max } \%>34$, tracer absorption into plasma was less than the corresponding lymphatic absorption (Fig. 7). This may be indicative of saturation of insulin absorption by blood at high degrees of adjuvant activity. Examination of the lymph:plasma partition ratio of tracer as a function of time revealed that lymphatic uptake appeared to lag behind that of plasma (Fig. 8). The effect of lymph flow rate on partitioning of tracer between plasma and lymph was investigated. No relationship between lymph flow rate and relative lymphatic uptake of tracer could be demonstrated.

The respective lymph:plasma partition ratios of insulin for the $0.024 \mathrm{M}$ and $0.15 \mathrm{M}$ concentrations of DIS did not differ significantly, implying that the decline in hypoglycemic effect seen at the higher concentration of adjuvant was not due to a decline in lymphatic targeting of insulin. However, the relative extent of tracer absorption between these two adjuvant concentrations differed by a factor of 2.8 for lymph and 2.5 for plasma, both being greater for the $0.024 \mathrm{M}$ concentration of DIS $(p<0.01$, $n \geq 5$ ).

\section{Protein Extraction Studies}

For the in vivo studies, a solution of adjuvant was introduced into a ligated rectal sac in situ and removed after $1 \mathrm{~h}$. A good relationship was

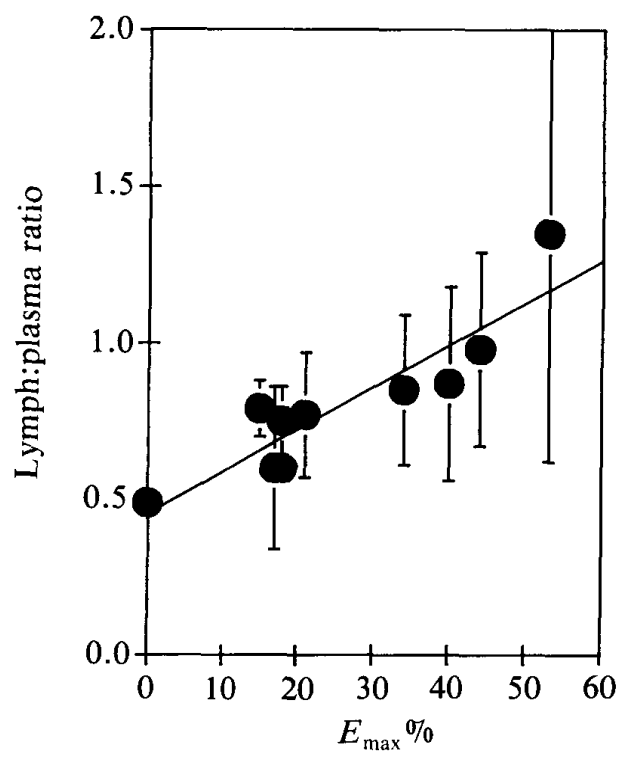

Fig. 6. Lymphatic Targeting of Insulin, Expressed as the Lymph: Plasma Ratios, as a Function of Adjuvant Activity $\left(E_{\max } \%\right)$

* RIA data reproduced with permission of the copy right owner, the American Pharmaceutical Association. 


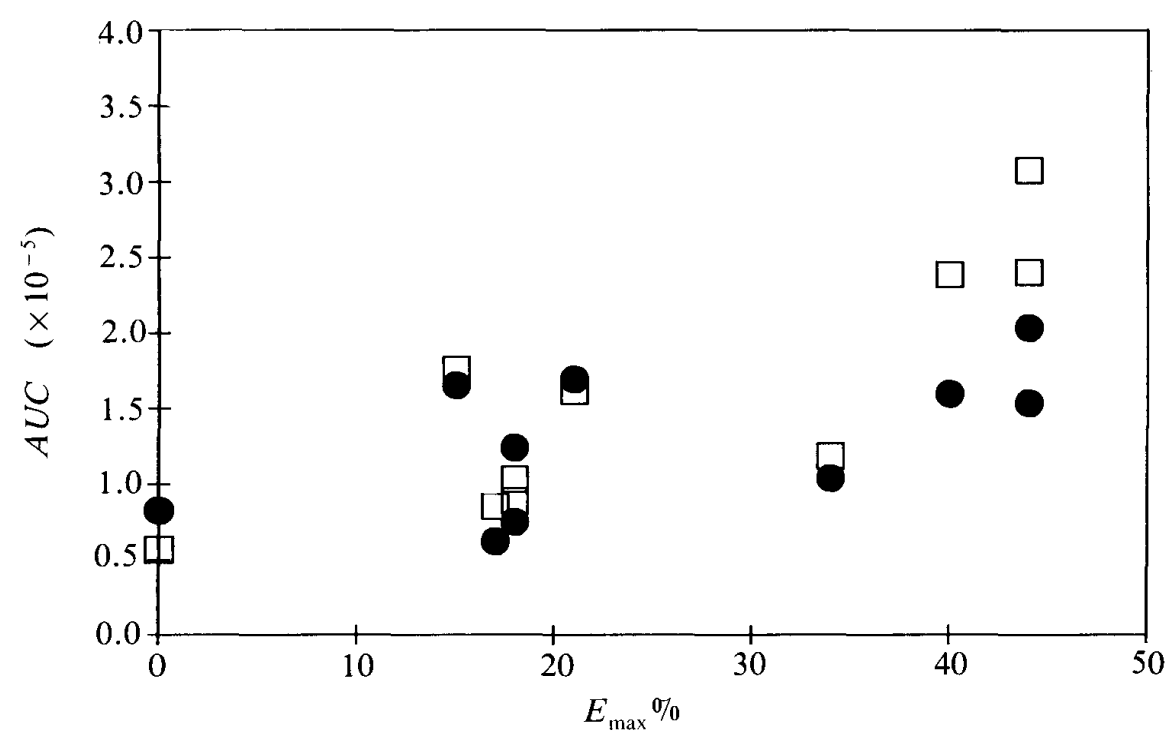

Fig. 7. Comparison of the $A U C$ for Insulin Absorbed into Lymph ( $\square$ ) and Plasma ( $)$ s. Time as a Function of Relative Adjuvant Activities $\left(E_{\max } \%\right)$

, plasma; $\square$, lymph.

found between total protein concentration in the dialyzed mucosal solutions and the corresponding, previously determined $E_{\max } \%$ values for the respective adjuvants (Fig. 9). Polyacrylamide gel-electrophoresis of the solutions gave banding patterns qualitatively similar to that of control, particularly with regard to the release of 2 high molecular weight proteins ( $>95500 \mathrm{Da}$ ) and

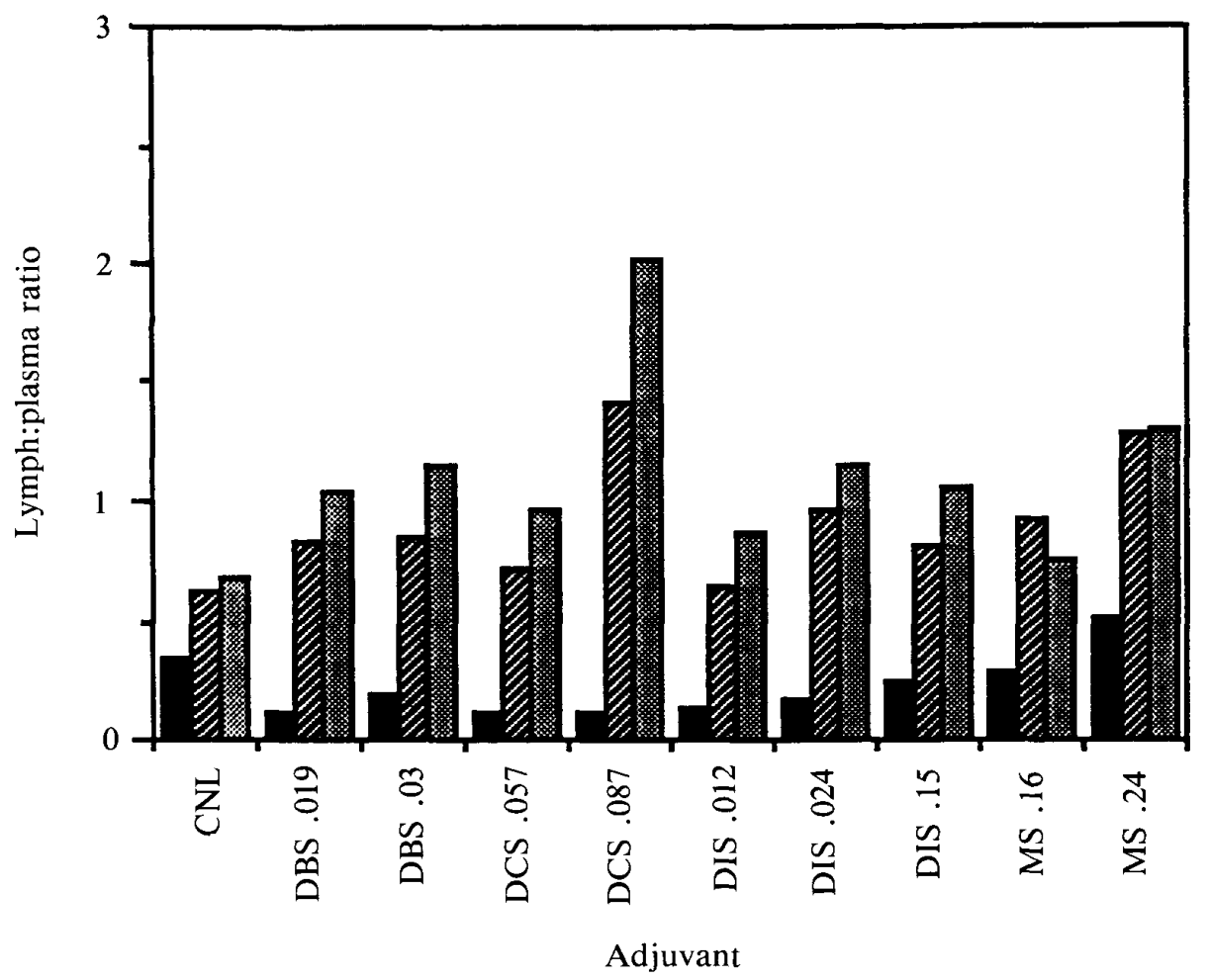

Fig. 8. Comparison of Lymph:Plasma Ratios for Insulin at 15, 45, and 90 min, Illustrating Lag Time in Lymphatic Absorption as Compared to Plasma Absorption

口, LPR 15; $\mathbb{Z}$, LPR 45; 爾, LPR 90. 


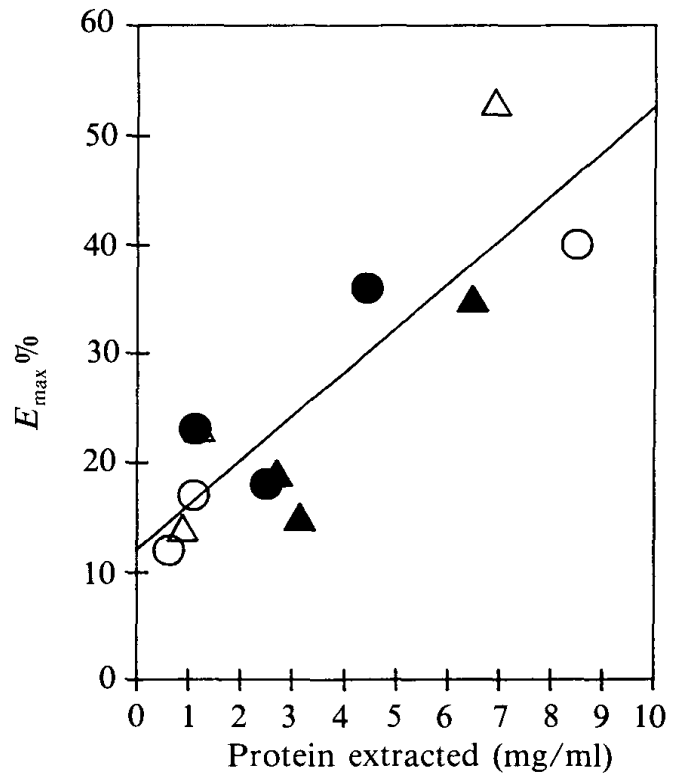

Fig. 9. Relationship of Adjuvant Activity $\left(E_{\max } \%\right)$ to Amount of Protein Extracted from the Rat Rectum in Situ O, MS;, DIS; $\triangle$, DCS; $\boldsymbol{\Lambda}$, DBS.

4 proteins with molecular weights of 94905 , 57199,46593 , and $30770 \mathrm{Da}$; for MS, the highest molecular weight proteins $(>95500 \mathrm{Da})$ did not resolve into two peaks, but instead yielded a single peak (Fig. 10). Sodium lauryl sulfate (SLS) and DIS $0.15 \mathrm{M}$, however, demonstrated significant increases $(p<0.05, n=3) v s$. both MS 0.25 $\mathrm{M}$ and control, in the intensity of the bands corresponding to a group of proteins with molecular weights ranging from about 11000 to 12000 Da. For control, these bands accounted for $2.6 \%$ \pm 1.29 of the total area of the scan; for MS 0.25 $\mathrm{M}, 1.16 \% \pm 1.37$; for DIS $0.15 \mathrm{M}, 9.19 \%$ \pm 1.37 ; and for SLS $5 \mathrm{mM}, 9.24 \% \pm 0.175$. Total protein extracted by DIS $0.15 \mathrm{M}$ was 3.01 $\pm 0.86 \mathrm{mg} / \mathrm{ml}$; for SLS $5 \mathrm{mM}, 5.09 \pm 0.59$ $\mathrm{mg} / \mathrm{ml}$, which was 1.69 times greater $(p<0.03$, $n=3$ ). For the in vitro studies, total protein concentration in the dialyzed mucosal solutions did not differ between control $(1502 \pm 5.5 \mu \mathrm{g} / \mathrm{ml})$ and treated specimens for MS $0.15 \mathrm{M}$ (1487 $\pm 28.53 \mu \mathrm{g} / \mathrm{ml}$ ), but showed a slight difference for DIS $0.025 \mathrm{M}(1583 \pm 17.52 \mu \mathrm{g} / \mathrm{ml})(n=3$, $p<0.01$, ANOVA). It was not possible to examine protein extraction by higher concentrations of DIS due to limited solubility of this compound in the physiological buffer solution.
A)

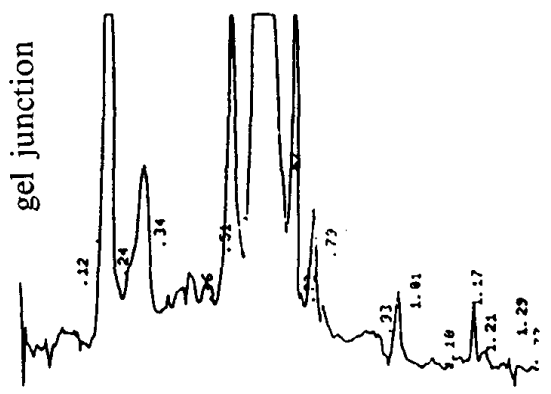

B)

C)

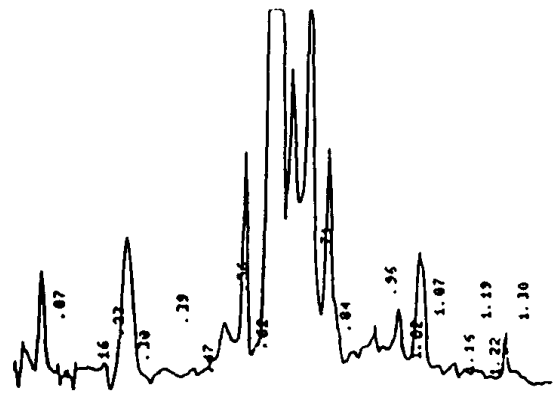

D)
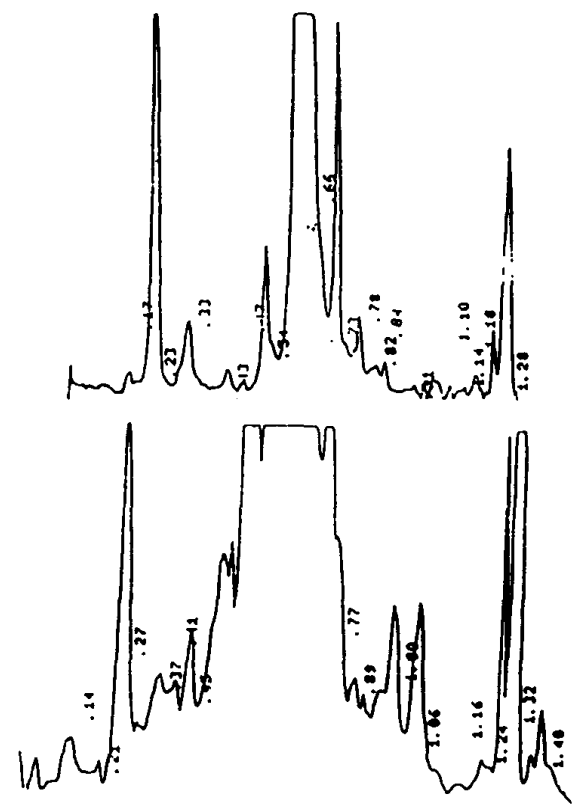

E)

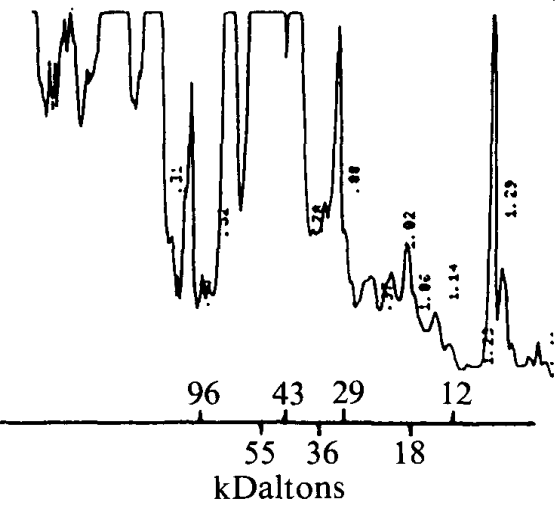

Fig. 10. The Protein Banding Patterns Obtained with Polyacrylamide Slab Electrophoresis of Proteins Extracted from the Rat Rectum in Situ in the Presence of Various Adjuvant Solutions

A) control, B) MS $0.25 \mathrm{M}$, C) DIS $0.15 \mathrm{M}$, D) SLS $5 \mathrm{~mm}$, E) rat plasma. The proteins were visualized with silver stain and the gels scanned on a Bio-Rad model 1650 densitometer. 


\section{Discussion}

We have demonstrated that the relative potencies of four salicylate analogs as adjuvant absorption promoters for insulin are related to the adjuvant hydrophobicity. Absorption-enhancing activity was closely related to the relative magnitude of activity on membrane ionic permeability in isolated molluscan neurons (Fig. 3). ${ }^{2)}$ The activities of salicylates in both of these systems is consistent with McLaughlin's studies ${ }^{15)}$ in which he concluded that the binding of salicylates to lipid bilayers involved primarily hydrophobic forces. Salicylate-induced enhancement of membrane permeability for positivelycharged ionic species correlated with the relative degree of accumulated negative charge on the membrane; ${ }^{15)}$ this in turn was directly related to the degree of association of salicylate with the membrane, which was a function of the relative lipophilicity of the salicylate analog. ${ }^{15}$ ) The results of a study by Nishihata et al. on the effects of various adjuvants on rectal absorption enhancement supported the relationship between adjuvant potency and degree of binding to the rectal membrane. ${ }^{16)}$ More recent work concerning the mechanism of absorption enhancement suggests that adsorption of salicylate to the membrane results in a perturbation in the interaction between membrane proteins and lipids; this in turn results in an increase in membrane fluidity which may be responsible for the consequent increase in membrane permeability. ${ }^{17,18)}$

A relationship was found between absorptionenhancing activity for insulin and relative degree of protein released in vivo (Fig. 9). Adjuvants whose absorption-enhancing activities are associated with major histological changes in epithelial membranes (i.e., anionic surfactants, bile salts) show correlations between degree of adjuvant activity and magnitude of protein released for both in vivo ${ }^{6,19)}$ and in vitro ${ }^{20)}$ preparations. For MS, in vitro protein release from everted rat rectal sacs was not significantly different from control. This is in agreement with the results of other investigators who studied the release of proteins by sodium salicylate from everted rectal sacs. ${ }^{6}$ In our study, the dependence of the relationship of salicylate ad- juvant activity to extent of protein release on an in vivo situation suggests the possibility that the protein release is dependent on an intact circulation. Enhancement of rectal absorption of macromolecules by adjuvants has been found to parallel exsorption of the test compounds from blood to rectal lumen. ${ }^{21)}$ This raises the possibility that in vivo release of proteins by salicylate adjuvants may partially be the result of enhanced exsorption of plasma proteins, which was supported by similarities between the electrophoretograms of the proteins released in vivo by salicylates and those of rat plasma.

The decline in adjuvant activity seen at 0.15 M DIS was associated with decreases in the amount of $\left[{ }^{125} I\right]$ insulin absorbed and the maximum hypoglycemic effect observed. In addition, it was associated with a high relative extraction, compared to MS, of a group of proteins ranging from approximately 11000 to $12000 \mathrm{Da}$ (Fig. 10). High concentrations of the adjuvant, diethyl maleate (DEM), produced similar declines in the enhanced intestinal absorption of cefmetazole, accompanied by extraction of protein thiols. ${ }^{5,7}$ The extraction of these proteins was postulated to produce a conformational change in the cytoskeletons of the absorptive cells, which may have increased the permeability of the paracellular pathway. ${ }^{22,23)}$ While the effects of protein extraction by DEM on paracellular permeability were not investigated, colonic absorption of cefmetazole was shown to normally occur predominantly via the paracellular route. ${ }^{5)}$ Salicylate-enhanced absorption of macromolecules is to believed to occur via the paracellular pathway. ${ }^{24,25)}$ Decreased absorption of both insulin ${ }^{1)}$ and cefmetazole, ${ }^{5)}$ drugs whose absorption is thought to occur paracellularly, were seen with increased concentrations of DIS ${ }^{1)}$ and DEM, ${ }^{5)}$ respectively; in both cases, the decreased absorption was associated with the extraction of specific proteins. It is not clear whether extraction of specific proteins at elevated concentrations of either adjuvant affects the permeability of the tight junctional region. Certain physicochemical characteristics of DIS relating to its superior abilities to solubilize protein may explain the singular occurrence of the decline in insulin absorption seen with this ad- 
juvant. Concentrations of DIS ranging from 0.1 to $0.3 \mathrm{M}$ produced notable changes on the gross structure of red cell ghost membranes, accompanied by the extraction of protein. ${ }^{26)}$ Similar concentration-effect phenomena, including extraction of cytoskeletal protein components, were seen on intestinal brush border membrane vesicles, suggesting that DIS may exert general effects on plasma membranes. ${ }^{2)}$ The ability of salts to solubilize proteins is generally a function of the reverse of the Hofmeister lyotropic series of ions, ${ }^{27)}$ making DIS an extremely efficient protein solubilizing agent. The presence of iodine in DIS greatly augments this protein saltingin effect. ${ }^{27)}$ The superior abilities of relatively low concentrations of DIS to solubilize membrane proteins, as compared to MS, was substantiated by our in vitro protein extraction studies.

The possibility that membrane damage is responsible for the decline in insulin absorption seen at $0.15 \mathrm{M}$ DIS cannot be excluded. However, differences in both the apparent mechanisms of adjuvant activity and degree and selectivity of protein extraction between DIS and SLS, an adjuvant noted for damage, make such an assertion questionable. This implies that the decline in adjuvant activity seen at $0.15 \mathrm{M}$ DIS may be more related to extraction of specific membrane protein components, as is thought to occur with DEM. ${ }^{5)}$ SLS $5 \mathrm{mM}$ produced a relative protein extraction profile comparable to DIS $0.15 \mathrm{M}$, but demonstrated nearly twice the relative total protein extraction. Other investigators have found this concentration of SLS to be associated with marked, but irreversible adjuvant activity. ${ }^{28)}$ This suggests loss of integrity of the membrane barrier, associated with extraction of protein, as being the primary mechanism of adjuvant action. SLS $34 \mathrm{~mm}$ has been associated with a time-dependent decline in absorption enhancement, which has been postulated to be due to tissue contracture to counteract fluid loss resulting from extensive damage to the membrane. This was confirmed by electron microscopy ${ }^{28)}$ and presumably is associated with extensive protein extraction. ${ }^{19)}$ Biochemical studies have related enhancement of paracellular absorption by salicylates to degree of extraction of reduced non-protein sulfhydryl (RNS); a similar relationship was not found for SLS, suggesting a mechanism of adjuvant activity different from that of salicylates. ${ }^{29)}$

The lymph:plasma partitioning ratio has been previously employed to quantitate the relative distribution of drug between plasma and lymph. ${ }^{30)}$ Our results have demonstrated that the relative degree of targeting of rectally absorbed insulin to the lymphatic compartment appears to be correlated with the $E_{\max } \%$ value. In addition, lymphatic absorption was found to lag behind that of blood. This could possibly be explained by the relatively sluggish perfusion of the gastrointestinal tract by lymph as opposed to that of blood, which has been found to be nearly 400 times greater. ${ }^{31)}$ It is possible that the increased hypoglycemic activity associated with a higher lymph:plasma absorption ratio for insulin is due to greater peripheral delivery of intact insulin following lymphatic absorption. ${ }^{32)}$ It is unlikely, however, that avoidance of hepatic first pass metabolism due to increased lymphatic absorption is responsible for the increased hypoglycemic activity, as much evidence indicates the liver to be the primary target organ for insulin activity. ${ }^{33)}$ It is also possible that the change in duration and magnitude of effect is not determined solely by increased lymphatic absorption of insulin but instead, simply reflects the systemic response to greater total delivery of insulin via both blood and lymph. ${ }^{14)}$

The ability of MS to target absorption of rectally delivered drugs to the lymphatic compartment has been previously described. ${ }^{34,35)}$ Suppression of competing blood absorption of drug by MS was postulated to be responsible for the selective lymphatic uptake. ${ }^{34)}$ Although experimental evidence was provided to support the theory of lymphatic targeting via suppression of competing blood absorption, no mechanism for this suppression was offered. ${ }^{34)}$ We did not study the relationship between adjuvant activity and suppression of competing blood absorption. However, comparisons of the area under the blood concentration-time curve ( $A U C$ 's) versus $E_{\max } \%$ for insulin tracer absorbed into lymph and blood, respectively, demonstrated a linear relationship for lymph whereas the similar relationship for blood indicated saturation at higher 
$E_{\max } \%$ values, which would be consistent with suppression of blood absorption.

A possible correlation between lymph flow rate and uptake of cefoxitin from the rectal compartment following administration with the adjuvant MS has been suggested, although it appeared that other factors were involved in determining degree of lymphatic uptake. ${ }^{35)} \mathrm{We}$ were not able to demonstrate a correlation between relative lymphatic absorption of insulin and lymph flow rate.

Certain ultrastructural differences in lymphatics and blood capillaries may be responsible for the selective uptake of insulin via lymph, following enhancement of absorption by salicylates. The lack of a basal lamina around lymphatic capillaries was considered to be of major functional importance in facilitating the transport of macromolecules into the lymph. ${ }^{36)}$ Further evidence indicates that macromolecular transport into lymph may be asymmetric, favoring movement from the interstitial space into the lymph. ${ }^{36)}$ This assertion is supported by findings that indicate the existence of a charge differential between the luminal and abluminal surfaces of the endothelial cells of the lymphatics. ${ }^{36)}$ Compared to the luminal surface, the abluminal surface is thought to possess a cationic charge, which would favor the transport of negatively charged proteins into lymph. The isoelectric $\mathrm{pH}$ of insulin is 5.3, which would render it negatively charged at physiologic $\mathrm{pH}$, thus favoring lymphatic absorption on the basis of charge. In addition to molecular size and charge, relative lymphatic uptake of a drug may be governed by its lipid solubility and affinity for cytoplasmic proteins, which play an important role in carrying lipid-soluble compounds into the portal system. ${ }^{37)}$

In summary, we have demonstrated that the relative potencies of salicylate adjuvants are related to the lipophilicity of the adjuvant. The decline in adjuvant activity seen at high concentrations of DIS may be associated with the high relative extraction of specific proteins of approximately 11 to $12 \mathrm{kDa}$. Finally, relative lymphatic targeting of rectally delivered insulin by salicylates appears to be correlated with magnitude of adjuvant activity.
Acknowledgments The authors wish to express sincere gratitude to Dr. Hiromitsu Kimura, for his patient and invaluable assistance in teaching us the Bollman technique. D.J.H. was the recipient of a Pharmaceutical Manufacturers Association 1988 Advanced Predoctoral Fellowship in Pharmaceutics. Kumaril Bhargava provided technical assistance.

\section{References}

1) D. J. Hauss and H. Y. Ando: The Influence of Concentration of Two Salicylate Derivatives on Rectal Insulin Absorption Enhancement, J. Pharm. Pharmacol., 40, 659-661 (1988).

2) H. Levitan and J. L. Barker: Salicylate: A StructureActivity Study of its Effects on Membrane Permeability, Science, 176, 1423-1425 (1972).

3) D. Riendeau, J. Lemaire, D. Maestracci and L. Lessard: Selective Release of Inner Core Proteins from Intestinal Microvillus Membrane by Lithium Diiodosalicylate, Mol. Cell. Biochem., 71, 45-52 (1986).

4) T. Nadai, M. Kume, A. Tatematsu and H. Sezaki: Drug-Induced Histological Changes and Its Consequences on the Permeability of the Small Intestinal Mucosa. II, Chem. Pharm. Bull., 23, 543-551 (1975).

5) T. Nishihata, T. Suzuka, A. Furuya, M. Yamazaki and A. Kamada: Influence of Diethyl Maleate-Induced Loss of Thiols on Cefmetazole Absorption from Ileal Loop of Rats, Chem. Pharm. Bull., 35, 2914-2922 (1987).

6) T. Nishihata, H. Tomida, G. Frederick, J. H. Rytting and T. Higuchi: Comparison of the Effects of Sodium Salicylate, Disodium Ethylenediaminetetraacetic Acid and Polyoxyethylene-23-Lauryl Ether as Adjuvants for the Rectal Absorption of Sodium Cefoxitin, J. Pharm. Pharmacol., 37, 159-163 (1985).

7) T. Nadai, M. Kume, A. Tatematsu and H. Sezaki: Effect of Tetracycline and Some Pharmaceutical Adjuvants on the Capillary and the Lymphatic Transport of Sulfanilic Acid from the Rat Small Intestine, Yakugaku Zasshi, 96, 545-549 (1976).

8) J. L. Bollman, J. C. Cain and J. C. Grindlay: Techniques for the collection of Lymph from the Liver, Small Intestine, or Thoracic Duct of the Rat, J. Clin. Med., 33, 1349-1352 (1948).

9) E. Touitou and A. Rubinstein: Targeted Enteral Delivery of Insulin to Rats, Int. J. Pharm., 30, 95-99 (1986).

10) R. J. Tallarida and R. B. Murray: "Manual of Pharmacologic Calculations with Computer Programs," Springer-Verlag Inc., New York, 1984, pp. 21-24.

11) T. Nishihata, J. H. Rytting, A. Kamada, T. Higuchi, M. Routh and L. Caldwell: Enhancement of Rectal Absorption of Insulin Using Salicylates in Dogs, J. Pharm. Pharmacol., 35, 148-151 (1983).

12) G. E. Peters, L. E. F. Hutchinson, R. Hyde, C. McMar- 
tin and S. B. Metcalfe: Effects of Sodium 5-Methoxysalicylate on Macromolecule Absorption and Mucosal Morphology in a Vascularly Perfused Rat Gut Preparation in Vivo, J. Pharm. Sci., 76, 857-861 (1987).

13) L. Caldwell, T. Nishihata, J. Fix, S. Selk, R. Cargill, C. R. Gardner and T. Higuchi: Absorption-promoting Adjuvants: Animal Studies on their Effects on Rectal Drug Absorption, Meth. Find. Exptl. Clin. Pharmacol., 6, 503-507 (1984).

14) C. Binder, T. Lauritzen, O. Faber and S. Pramming: Insulin Pharmacokinetics, Diabetes Care, 7, 188-199 (1984).

15) S. McLaughlin: Salicylates and Phospholipid Bilayer Membranes, Nature (London), 243, 234-236 (1973).

16) T. Nishihata, J. H. Rytting and T. Higuchi: Enhanced Rectal Absorption of Theophylline, Lidocaine, Cefmetazole, and Levodopa by Several Adjuvants, $J$. Pharm. Sci., 71, 865-868.

17) H. Kajii, T. Horie, M. Hayashi and S. Awazu: Fluorescence Study on the Interaction of Salicylate with Rat Small Intestinal Epithelial Cells: Possible Mechanism for the Promoting Effects of Salicylate on Drug Absorption in Vivo, Life Sci., 37, 523-530 (1985).

18) H. Kajii, T. Horie, M. Hayashi and S. Awazu: Effects of Salicylic Acid on the Permeability of the Plasma Membrane of the Small Intestine of the Rat: A Fluorescence Spectroscopic Approach to Elucidate the Mechanism of Promoted Drug Absorption, J. Pharm. Sci., 75, 475-478 (1986).

19) K. Nakanishi, S. Miyazaki, M. Masada and T. Nadai: Effect of Pharmaceutical Adjuvants on the Rectal Permeability of Drugs. I. Effect of Pharmaceutical Additives on the Permeability of Sulfaguanidine from the Rat Rectum, Yakugaku Zasshi, 102, 1133-1140 (1982).

20) D. A. Whitmore, L. G. Brookes and K. P. Wheeler: Relative Effects of Different Surfactants on Intestinal Absorption and the Release of Proteins and Phospholipids from the Tissue, J. Pharm. Pharmacol., 31, 277-283 (1979).

21) K. Nakanishi, M. Masada and T. Nadai: Effect of Pharmaceutical Adjuvants on the Rectal Permeability to Drugs IV. Effect of Pharmaceutical Adjuvants on the Rectal Permeability to Macromolecular Compounds in the Rat, Chem. Pharm. Bull., 32, 1628-1632 (1984).

22) I. Meza, M. Sabanero, E. Stefani and M. Cereijido: Occluding Junctions in MDCK Cells: Modulation of Transepithelial Permeability by the Cytoskeleton, $J$. Cell. Biochem., 18, 407-421 (1982).

23) E. E. Schneeberger and R. D. Lynch: Tight Junctions: Their Structure, Composition, and Function, Circ. Res., 55, 723-733 (1984).

24) T. Nishihata, M. Miyake, H. Takahata and A. Kamada: The Effect of Adjuvants on the Colonic Absorp- tion of Cefmetazole and [Asu $\left.{ }^{1,7}\right]$-Eel Calcitonin in Rats: Concentration Dependent Absorption Pathways, Int. J. Pharm., 33, 89-97 (1986).

25) A. Martinez-Paloma, I. Meza, G. Beaty and M. Cereijida: Experimental Modulation of Occluding Junction in a Cultured Transporting Epithelium, J. Cell. Biol., 87, 736-745 (1980).

26) V. T. Marchesi and E. P. Andrews: "Glycoproteins: Isolation from Cell Membranes with Lithium Diiodosalicylate, Science, 174, 1247-1248 (1971).

27) Dr. R. Robinson and W. P. Jencks: The Effect of Concentrated Salt Solutions on the Activity Coefficient of Acetyltetraglycine Ethyl Ester, J. Am. Chem. Soc., 87, 2470-2479 (1965).

28) T. Nadai, R. Kondo, A. Tatematsu and H. Sezaki: Drug-Induced Histological Changes and its Consequences on the Permeability of the Small Intestinal Mucosa. I. EDTA, Tetracycline, and Sodium Laurylsulfate, Chem. Pharm. Bull., 20, 1139-1144 (1972).

29) T. Nishihata, H. Takahata and A. Kamada: Possible Mechanism Regulating Barrier Function of Rat Intestinal Mucosa Against Permeation of Cefmetazole, a Hydrophilic Drug, Pharm. Res., 2, $307-309$ (1985).

30) D. G. Garlick and E. M. Renkin: Transport of Large Molecules from Plasma to Interstitial Fluid and Lymph in Dogs, Am. J. Physiol., 219, 1595-1605 (1970).

31) E. J. Reininger and L. A. Sapirstein: Effect of Digestion on Distribution of Blood Flow in the Rat, Science, 126, 1176 (1957).

32) J. Tiran, L. I. Avruch and A. M. Albisser: A Circulation and Organs Model for Insulin Dynamics, Am J. Physiol., 237, E331-E339 (1979).

33) W. A. Ritschel and G. B. Ritschel: Rectal Administration of Insulin, Meth. and Find. Exptl. Clin. Pharmacol., 6, 513-529 (1984).

34) T. Nishihata, K. Yasui, M. Yamazaki and A. Kamada: Effect of Adjuvants on the Rectal Absorption and Lymphatic Uptake of Pepleomycin in Rats, J. Pharmacobio-Dyn., 7, 278-285 (1984).

35) T. Nishihata, S. Kim, A. Kamada, G. Frederick, M. Dillsaver and T. Higuchi: Lymphatic Transport of Sodium Cefoxitin in the Presence of Sodium 5-Methoxysalicylate after Injection into Rat Rectal Connective Tissue, Femoral Muscle and Femoral Vein, $J$. Pharm. Pharmacol., 37, 509-511 (1984).

36) C. C. C. O'Morchoe and P. J. O'Morchoe: Differences in Lymphatic and Blood Capillary Permeability: Ultrastructural-Functional Correlations, Lymphology, 20, 205-209 (1987).

37) T. Noguchi, K. Taniguchi, T. Yoshifuji, S. Muranishi and H. Sezaki: Lymphatic Transport of Griseofulvin in the Rat and the Possible Factors Determining the Extent of Lymphatic Absorption, Chem. Pharm. Bull., 25, $2231-2238$ (1977). 